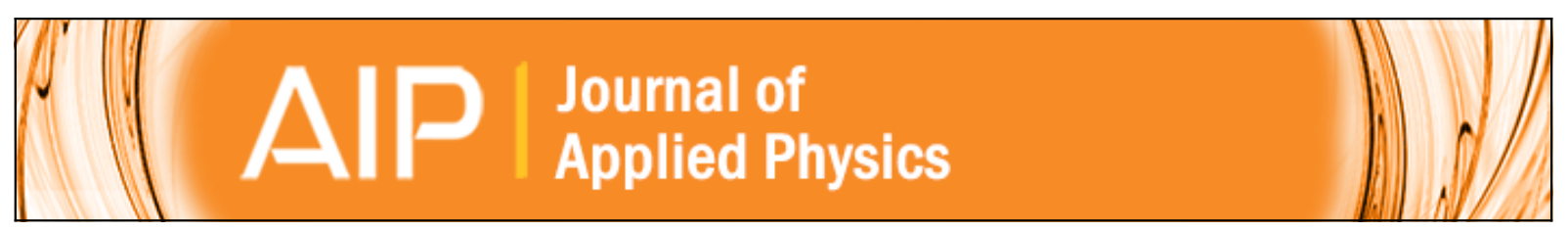

\title{
On effective surface recombination parameters
}

Keith R. Mclntosh and Lachlan E. Black

Citation: Journal of Applied Physics 116, 014503 (2014); doi: 10.1063/1.4886595

View online: http://dx.doi.org/10.1063/1.4886595

View Table of Contents: http://scitation.aip.org/content/aip/journal/jap/116/1 ?ver=pdfcov

Published by the AIP Publishing

\section{Articles you may be interested in}

Analysis of sub-stoichiometric hydrogenated silicon oxide films for surface passivation of crystalline silicon solar cells

J. Appl. Phys. 112, 054905 (2012); 10.1063/1.4749415

Investigation of electrical shading effects in back-contacted back-junction silicon solar cells using the twodimensional charge collection probability and the reciprocity theorem

J. Appl. Phys. 109, 024507 (2011); 10.1063/1.3524506

Analyzing the effects of front-surface fields on back-junction silicon solar cells using the charge-collection probability and the reciprocity theorem

J. Appl. Phys. 103, 054507 (2008); 10.1063/1.2887991

Accurate measurement of extremely low surface recombination velocities on charged, oxidized silicon surfaces using a simple metal-oxide-semiconductor structure

Appl. Phys. Lett. 90, 042104 (2007); 10.1063/1.2434172

Surface recombination velocity of phosphorus-diffused silicon solar cell emitters passivated with plasma enhanced chemical vapor deposited silicon nitride and thermal silicon oxide

J. Appl. Phys. 89, 3821 (2001); 10.1063/1.1350633

AlP $\left.\right|_{\text {Letters }} ^{\text {Applied Physics }}$

is pleased to announce Reuben Collins as its new Editor-in-Chief

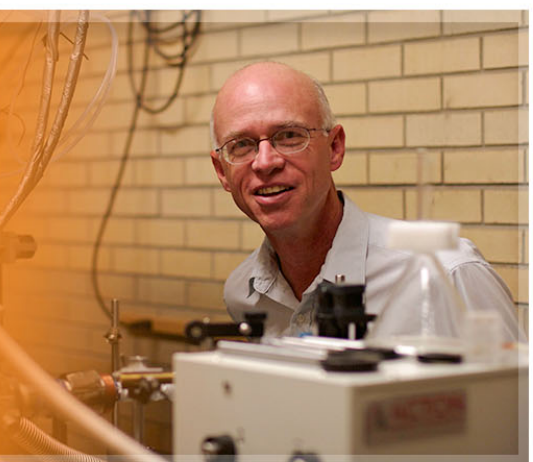




\title{
On effective surface recombination parameters
}

\author{
Keith R. McIntosh ${ }^{1}$ and Lachlan E. Black ${ }^{2}$ \\ ${ }^{1}$ PV Lighthouse, Coledale NSW 2515, Australia \\ ${ }^{2}$ College of Engineering and Computer Science, Australian National University, Canberra ACT 2600, Australia
}

(Received 11 April 2014; accepted 18 June 2014; published online 7 July 2014)

\begin{abstract}
This paper examines two effective surface recombination parameters: the effective surface recombination velocity $S_{\text {eff }}$ and the surface saturation current density $J_{O s}$. The dependence of $S_{\text {eff }}$ and $J_{O S}$ on surface charge $Q$, surface dopant concentration $N_{s}$, and interface parameters is derived. It is shown that for crystalline silicon at $300 \mathrm{~K}$ in low-injection, $S_{\text {eff }}$ is independent of $N_{s}$ only when $Q^{2} / N_{s}<1900 \mathrm{~cm}$ in accumulation and $Q^{2} / N_{s}<1600 \mathrm{~cm}$ in depletion; otherwise $S_{\text {eff }}$ increases with $N_{s}$. These conditions are rarely satisfied in undiffused wafers but sometimes satisfied in heavily diffused wafers when coated with lowly charged films. Under the same conditions, $J_{O S}$ is independent of $N_{s}$ when $Q^{2} / N_{s}>1.5 \times 10^{7} \mathrm{~cm}$ for accumulation and $Q^{1.85} / N_{s}>1.5 \times 10^{6} \mathrm{~cm}$ for inversion. These conditions are commonly satisfied in undiffused wafers but rarely in diffused wafers. We conclude that for undiffused silicon, $J_{O S}$ is superior to the conventional $S_{\text {eff }}$ as a metric for quantifying the surface passivation, whereas for diffused silicon, the merit in using $J_{O s}$ or $S_{\text {eff }}$ (or neither) depends on the sample. Experimental examples are given that illustrate the merits and flaws of $J_{O s}$ and $S_{\text {eff. }}$. 2014 AIP Publishing LLC. [http://dx.doi.org/10.1063/1.4886595]
\end{abstract}

\section{INTRODUCTION}

The passivation of a semiconductor surface is commonly characterised by an "effective surface recombination velocity." This parameter combines a multitude of interface properties, making it simple to quantify and compare surface treatments.

The effective surface recombination velocity $S_{\text {eff }}$ is defined by the equation

$$
J_{\text {rec }}=q U_{s}=q S_{e f f} \Delta n_{d},
$$

where $J_{\text {rec }}$ is the current density that flows into the surface to recombine, $q$ is the charge of an electron, $U_{s}$ is the recombination rate at the surface in $\mathrm{cm}^{-2} \mathrm{~s}^{-1}$, and $\Delta n_{d}$ is the excess carrier concentration "near" the surface. As illustrated in Figure 1, the subscript $d$ refers to the distance from the surface where the carrier concentrations are negligibly affected by surface charge. Usually, this distance is sufficiently close to the surface $s$ that the difference between the doping concentrations at $s$ and $d$ is insignificant.

The merit in using a single parameter to quantify surface recombination is compromised when that parameter depends on properties unrelated to the passivation layer, such as the surface dopant concentration $N_{S}$ or the excess carrier concentration away from the surface $\Delta n_{d}$. This is unfortunately the case for $S_{\text {eff }}$ under most practical conditions. There are two reasons.

The first reason is that when surface charge is significant, $S_{e f f}$ depends on $N_{s}$ and $\Delta n_{d \cdot}{ }^{1-4}$ In fact, for undiffused silicon wafers, very little surface charge is required to introduce this dependence (see Sec. VI). Without an appreciation of this effect, one might easily misinterpret the many observations of $S_{\text {eff }}$ increasing with $N_{s}$ [Refs. 5-12] as necessarily being caused by an underlying change to the interface (e.g., more defect states or increased capture cross sections).
The second reason is that $S_{\text {eff }}$ necessarily depends on $\Delta n_{d}$ over a large range of $\Delta n_{d}$ as a result of recombination carrier statistics. This dependence arises when $U_{s}$ transitions from being limited by the supply of minority carriers to being limited by the supply of both carrier species, as is evident from an examination of the Shockley-Read-Hall (SRH) equation for recombination through defect states. ${ }^{1,13,14}$ The resulting variation in $S_{\text {eff }}$ has a complicated dependence on the doping concentration, the electron and hole capture cross sections, and the energy of the defect. Under certain conditions, the dependence of $S_{\text {eff }}$ on $\Delta n_{d}$ is negligible; this is the case for defects not near the band edges when the majority carrier capture cross section greatly exceeds the minority

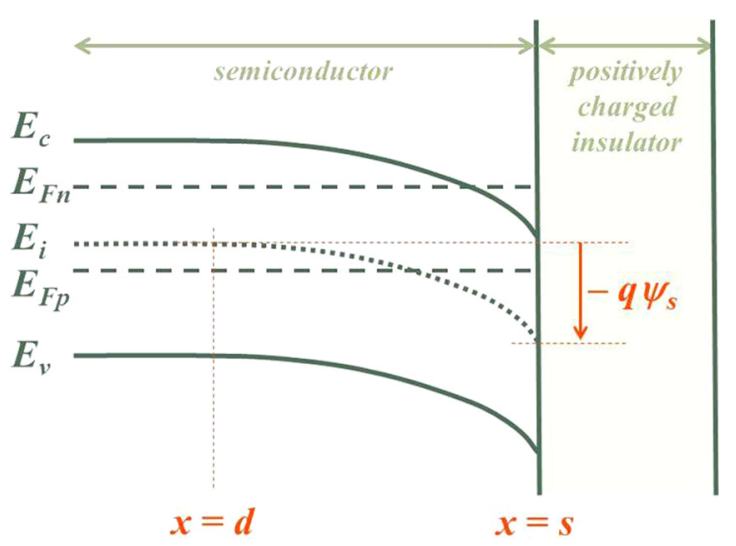

FIG. 1. Energy band diagram of a semiconductor-insulator interface under illumination, where the semiconductor is n-type and the insulator is positively charged. The diagram shows the energy of the conduction band $E_{c}$, the valence band $E_{v}$, the intrinsic Fermi level $E_{i}$, as well as the quasi-Fermi energy of electrons $E_{F n}$ and holes $E_{F p}$. The location $x=s$ represents the surface of the semiconductor (i.e., the interface) and $x=d$ is the point at which the energy bands are unaffected by charge in the insulator or at the interface. The potential is defined to be zero at $E_{i d}$ (that is, $E_{i}$ at $x=d$ ) and therefore band bending at the surface is given by $-q \psi_{s}=\left(E_{i d}-E_{i s}\right)$. 
carrier capture section. Under other conditions, $S_{\text {eff }}$ varies over a range of $\Delta n_{d}$ that is of no practical relevance, such as can occur when surface charge is large. But under many conditions, the dependence of $S_{\text {eff }}$ on $\Delta n_{d}$ is both significant and practically relevant, particularly when the minority carrier capture cross section exceeds the majority carrier cross section.

In this paper, we first introduce an alternative to $S_{\text {eff }}$ called the surface saturation current density $J_{0 s}$, which is analogous to the well-known emitter saturation current density $J_{0 e}$. Like $S_{\text {eff, }} J_{0 s}$ suffers from being dependent on $\Delta n_{d}$ and $N_{s}$ due to $\mathrm{SRH}$ recombination statistics. It also depends on $N_{s}$ when the surface charge is small. However, unlike $S_{e f f}, J_{0 s}$ is independent of $N_{s}$ and $\Delta n_{d}$ when the surface charge is large, making it a superior metric for quantifying surface passivation.

After defining $J_{O S}$, we next derive $S_{\text {eff }}$ and $J_{O S}$ in terms of $\mathrm{SRH}$ recombination parameters for various special cases. For each case we show the ranges of surface charge and $N_{s}$ over which $S_{\text {eff }}$ and $J_{O S}$ are independent of $N_{s}$. We can therefore conclude when it is preferable to use $S_{\text {eff }}$ rather than $J_{O s}$, and vice versa. We find that for undiffused silicon, it is preferable to use $J_{O S}$ under most practical conditions, whereas for diffused silicon, the benefit of using $S_{\text {eff }}$ or $J_{O S}$ (or neither) depends on the sample; we give examples of both.

\section{DEFINITION OF $J_{O s}$}

The surface saturation current density $J_{0 s}$ is analogous to the emitter saturation current density $J_{O e}$ (Ref. 15) and is defined by the equation

$$
J_{r e c}=q U_{s}=J_{0 s}\left[\frac{p_{s} n_{s}}{p_{0 s} n_{0 s}}-1\right] .
$$

Equation (2) relates the recombination current to the concentration of holes and electrons in steady state $(p$ and $n)$ and at equilibrium $\left(p_{0}\right.$ and $\left.n_{0}\right)$. As depicted in Figure 1 , the subscript $s$ refers to the actual surface of the semiconductor (i.e., the semiconductor-insulator interface), not some position near the surface.

The square-root of the product of the equilibrium concentrations is often called the effective intrinsic carrier concentration, $n_{i e}=\sqrt{p_{0 s} n_{0 s}}$. This parameter is dependent on the net doping concentration, $N=N_{A}-N_{D}$, due to band gap narrowing $(\mathrm{BGN})$ and degeneracy. ${ }^{8}$ It is equal to $n_{i}$ when $N=0$, that is, when the semiconductor is intrinsic. For the equations that follow, we assume that $n_{i e}$ is the same at $s$ and $d$ (i.e., that $p_{0 s} n_{O s}=p_{0 \mathrm{~d}} n_{0 \mathrm{~d}}$ ), and Appendix A describes how those equations can be rewritten when $p_{0 s} n_{0 s} \neq p_{0 \mathrm{~d}} n_{0 \mathrm{~d}}$.

The first term in the square brackets of Eq. (2) relates to the separation of quasi-Fermi levels at the surface $V_{s}$. When Boltzmann statistics apply (i.e., in non-degenerate semiconductors), the equation can be rewritten in the familiar form of the diode equation

$$
J_{r e c}=J_{0 s}\left[\exp \left(\frac{V_{s}}{V_{T}}\right)-1\right],
$$

where $V_{T}$ is the thermal voltage and equal to $k T / q, k$ is Boltzmann's constant, and $T$ is the absolute temperature.
It is not immediately apparent how or when $J_{O s}$ offers an advantage over $S_{\text {eff }}$ since its definition by Eq. (2) is more complicated than Eq. (1). It will be shown, however, that for undiffused silicon, $J_{O S}$ is independent of $N_{S}$ under most practical conditions, whereas $S_{\text {eff }}$ is constant only when there is an impractically small surface charge and in low injection. The $J_{O S}$ also has the advantage that for "transparent" emitters, it relates directly to the emitter saturation current density $J_{O e}$ of a diffused surface.

\section{DEPENDENCE OF $J_{O S}$ ON SRH PARAMETERS}

To show how $J_{O S}$ depends on defect parameters, we begin with the SRH equation for a single defect energy, ${ }^{1}$

$$
U_{S}=\frac{p_{s} n_{s}-n_{i e}^{2}}{\frac{p_{s}+p_{1}}{S_{n 0}}+\frac{n_{s}+n_{1}}{S_{p 0}}} .
$$

The terms $p_{1}$ and $n_{1}$, which incorporate the thermal emission of carriers from the defect energy, are given by $n_{1}=n_{i e}$ $\exp \left[\left(E_{t}-E_{i}\right) / k T\right]$ and $p_{1}=n_{i e} \exp \left[\left(E_{i}-E_{t}\right) / k T\right]$, where $E_{t}$ is the energy of the defect state. The parameters $S_{n 0}$ and $S_{p 0}$ are the surface recombination velocity parameters of electrons and holes, respectively, and given by $S_{n 0}=v_{t h n} N_{i t} \sigma_{n}$ and $S_{p 0}=v_{t h p} N_{i t} \sigma_{p}$, where $v_{t h n}$ and $v_{t h p}$ are the thermal velocity of electrons and holes, $\sigma_{n}$ and $\sigma_{p}$ are the capture cross sections of electrons and holes, and $N_{i t}$ is the defect density in $\mathrm{cm}^{-2}$.

Equation (4) is then rearranged to

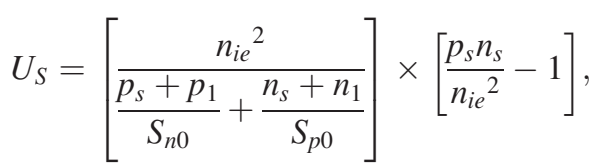

and combined with Eq. (2) to give $J_{O S}$ for a single SRH defect level

$$
J_{0 s}=\frac{q n_{i e}^{2}}{\frac{p_{s}+p_{1}}{S_{n 0}}+\frac{n_{s}+n_{1}}{S_{p 0}}} .
$$

Equation (6) can be extended to a continuum of defect energies in the manner of Refs. 13 and 16. Appendix B describes how the accommodation of this continuum need not involve numerical integration across the band gap.

Thus, like $U_{s}, J_{O s}$ depends on the equilibrium and steady-state carrier concentrations at the surface $s$. We now describe how they depend on surface doping and surface charge.

\section{SIMPLIFICATIONS TO THE SRH EQUATION}

To illustrate the difference between $S_{\text {eff }}$ and $J_{O s}$, we apply the SRH equation to n-type silicon and impose the restrictions that $n_{\mathrm{s}} \gg n_{1}$, and $\left(n_{s}+n_{l}\right) / S_{p 0} \gg\left(p_{s}+p_{1}\right) / S_{n 0}$. This describes the situation where the defect is not very close to the band edges, where the sample is not highly depleted or in mild inversion, and where there is no great asymmetry in the capture cross sections. We also impose the restriction 
that $p_{s} n_{s} \gg n_{i e}{ }^{2}$, which is valid in all but very low injection. (This latter restriction is unnecessary in the following equations for $J_{O S}$ but it does simplify $S_{\text {eff. }}$ ) The aforementioned conditions arise regularly, particularly when the doping or surface charge is high.

Under these assumptions Eq. (4) simplifies to

$$
U_{s}=S_{p 0} p_{s} .
$$

$S_{\text {eff }}$ for this simplified SRH equation is found by substituting Eq. (7) into Eq. (1) and by insisting that the equilibrium minority carrier concentration at $d$ is negligible, and hence

$$
\begin{gathered}
n_{d}=N_{D}+\Delta n_{d}, \\
p_{d} \sim \Delta n_{d},
\end{gathered}
$$

which therefore gives

$$
S_{e f f}=S_{p 0} \frac{p_{s}}{p_{d}}
$$

Thus, under the aforementioned conditions, $S_{\text {eff }}$ equals $S_{p 0}$ only when $p_{s}=p_{d}$, that is, when there is negligible band bending at the surface. When positive surface charge is applied, $p_{s}<p_{d}$, making $S_{\text {eff }}<S_{p 0}$. The converse also occurs for negative charge although either a very small or high negative charge is required to satisfy the aforementioned assumptions. It will be shown in Sec. $\mathrm{V}$ that when the charge is high enough, $S_{\text {eff }}$ becomes proportional to $n_{d}$.

$J_{O S}$ for the simplified SRH conditions is found by substituting Eq. (7) into Eq. (2) to give

$$
J_{0 s}=\frac{q S_{p 0} n_{i e}^{2}}{n_{s}}
$$

where the value of this equation will become apparent once the dependence of $n_{s}$ and $p_{s}$ on charge has been described.

\section{DEPENDENCE OF $S_{\text {eff }}$ AND $J_{o s}$ ON DOPING AND SURFACE CHARGE}

The relationship between the carrier concentrations, band bending, and surface charge is governed by the wellknown equation $^{17}$

$$
p_{s}-p_{d}+n_{s}-n_{d}+\left(N_{A}-N_{D}\right) \frac{\psi_{s}}{V_{T}}=\frac{Q^{2}}{2 q \epsilon_{S i} V_{T}},
$$

where $\psi_{s}$ is the surface potential relative to $\psi_{d}=0$ (see Figure 1$), \varepsilon_{S i}$ is the permittivity of the semiconductor, and $Q$ is the charge within the semiconductor that is invoked to balance the sum of the charge in the insulator and at the insulator-semiconductor interface. ${ }^{16}$ The dimensions for $Q$ are $\mathrm{C} / \mathrm{cm}^{2}$. Equation (12) assumes that the quasi-Fermi levels between $s$ and $d$ are flat.

The carrier concentrations can also be related to the band bending $\psi_{s}$. When Boltzmann statistics are applicable and the quasi-Fermi levels are flat, $n_{s}$ and $p_{s}$ can be written as

$$
n_{s}=n_{d} \exp \left(\frac{\psi_{s}}{V_{T}}\right) \text { and }
$$

$$
p_{s}=p_{d} \exp \left(-\frac{\psi_{s}}{V_{T}}\right)
$$

and hence

$$
p_{s} n_{s}=p_{d} n_{d}
$$

(More generally, the exponential functions can be replaced with the Fermi-Dirac function, as is required when Boltzmann statistics do not apply.)

Thus, in the manner described by Girisch et al. ${ }^{16}$ and its extension by Aberle et al., ${ }^{13}$ one can combine Eqs. (4) and (12)-(14) to determine $p_{s}$ and $n_{s}$, and then determine $S_{\text {eff }}$ with Eq. (1) and $J_{O s}$ with Eq. (2), or when applicable, with Eqs. (10) or (11). Girisch et al. show how to account for interface charge, which is also dependent on $\psi_{s}{ }^{16}$

Since Eq. (12) is implicit, it must be solved numerically except when certain special cases are applicable. Solving Eq. (12) numerically is not difficult but it is instructive to derive explicit equations for $U_{s}$. We consider four special cases, all of which lead to a simple analytical equation for $U_{s}$ and therefore for $S_{e f f}$ and $J_{0 s}$. The main purpose of deriving these analytical equations is to help explain when $S_{\text {eff }}$ and $J_{0 s}$ are independent of $N_{D}$.

The derivation of $S_{\text {eff }}$ for the four special cases is similar to those derived by Kuhlmann, ${ }^{2}$ Brody and Rohatgi, ${ }^{3}$ and Steingrube et al., ${ }^{4}$ except that it includes the extensions of including Case 2 and of removing the requirement that there be either low- or high-injection in the remaining cases (though they remain predicated on the validity of Eq. (7)).

The four special cases are these

(1) $Q$ is negligibly small, and hence $\psi_{s} \sim 0$ and

$$
n_{s}=n_{d}
$$

(2) $Q$ is sufficiently small that only the $k=2$ term in Eq. (C2) is significant (see derivation in Appendix C), and the sample is in low injection, $n_{d} \sim N_{D}$, and hence

$$
n_{s}=n_{d} \exp \left[\frac{Q}{\sqrt{k T \epsilon_{S i} N_{D}}}\right] .
$$

(3) $Q$ is sufficiently positive that the LHS of Eq. (12) equals $n_{s}$ (i.e., $n_{s} \gg n_{d}, p_{s}, p_{d}$ ), and hence

$$
n_{s}=\frac{Q^{2}}{2 k T \epsilon_{S i}} .
$$

(4) $Q$ is sufficiently negative that the LHS of Eq. (12) equals $p_{s}$ (i.e., $\left.p_{s} \gg n_{s}, n_{d}, p_{d}\right)$, and hence

$$
p_{s}=\frac{Q^{2}}{2 k T \epsilon_{S i}} .
$$

Notice that in Cases 3 and $4, n_{s}$ and $p_{s}$ are independent of the doping $N_{D}$ and the excess carriers $\Delta n_{d}$ at $d$. In fact, for undiffused silicon, either Case 3 or 4 holds in most practical situations. (Also note that for Case 4, the inversion of the surface polarity converts Eqs. (7), (10), and (11) into $U_{s}=S_{n 0} n_{s}$, $S_{e f f}=S_{n 0} n_{s} / p_{d}$, and $J_{O s}=q S_{n 0} n_{i e}^{2} / p_{s}$, respectively.) 
TABLE I. Solutions for $S_{e f f}$ and $J_{O s}$ in terms of SRH recombination parameters, surface charge $Q$ and electron concentration at $d\left(n_{d}=N_{D}+\Delta n_{d}\right)$ for n-type silicon under the restrictions required for Eq. (7) to be valid. With the exception of Case 2, the equations for $S_{\text {eff }}$ have been derived previously for low injection $\left(n_{d}=N_{D}\right)$ and $n_{i s}=n_{i d}=n_{i e}\left[\right.$ Refs. 2-4]; Brody and Rohatgi also derive Case 4 for high injection. ${ }^{3}$

\begin{tabular}{llcc}
\hline \hline Case & \multicolumn{1}{c}{ Approximation } & $S_{\text {eff }}$ & $J_{0 S}$ \\
\hline 1 & Negligible $Q$ such that $n_{s}=n_{d}$ & $S_{p 0}$ & $q \frac{S_{p 0}}{n_{d}} n_{i e}^{2}$ \\
2 & Low $Q$, low injection & $S_{p 0} \exp \left[\frac{-Q}{\left.\sqrt{k T \epsilon_{S i} N_{D}}\right]}\right.$ & $-Q \frac{S_{p 0}}{n_{d}} \exp \left[\frac{-Q}{\left.\sqrt{k T \epsilon_{S i} N_{D}}\right] n_{i e}^{2}}\right.$ \\
3 & Large positive $Q$ (strong accumulation) & $S_{p 0} n_{d} \frac{2 k T \epsilon_{S i}}{Q^{2}}$ & $q S_{p 0} \frac{2 k T \epsilon_{S i}}{Q^{2}} n_{i e}^{2}$ \\
4 & Large negative $Q$ (strong inversion) & $S_{n 0} n_{d} \frac{2 k T \epsilon_{S i}}{Q^{2}}$ & $q S_{n 0} \frac{2 k T \epsilon_{S i}}{Q^{2}} n_{i e}^{2}$ \\
\hline \hline
\end{tabular}

Section VI will define when these four special cases are valid, but before then, we examine how they simplify $S_{\text {eff }}$ and $J_{0 s}$. Table I lists the analytical expressions for $S_{\text {eff }}$ and $J_{0 s}$ derived for each case. There are many other ways to manipulate the expressions; those given in Table I highlight the dependencies on $N_{D}$ and $Q$.

In regards to $S_{\text {eff }}$, and keeping in mind that the derivations relied on the validity of Eq. (7), the most important features in Table I are these

- When $Q$ and therefore band-bending is negligible, $S_{\text {eff }}$ equals $S_{p o}$. Under this scenario (which rarely occurs for silicon), the common approach of employing $S_{\text {eff }}$ to define surface passivation is substantiated because it is independent of $n_{d}$.

- When $Q$ is not negligible, $S_{\text {eff }}$ is linearly related to $S_{p 0}$ (or $S_{n 0}$ ) but is also dependent on $n_{d}$ and hence the doping and excess carriers at $d$.

- When $Q$ is large, $S_{\text {eff }}$ is proportional to $S_{p 0 n d}$ (for positive $Q$ ) or $S_{n 0 \cdot n d}$ (for negative $Q$ ).

It is evident, therefore, that unless $Q$ is negligible, $S_{\text {eff }}$ should not be stated without also stating $n_{d}$; i.e., without stating the bulk doping and the excess carriers at $d$. This limits the applicability of using a measured $S_{\text {eff }}$ as a boundary condition for simulating surfaces that do not have the same $n_{d}$.

In some experimental studies, ${ }^{5-12} S_{\text {eff }}$ is found to increase with $N_{D}$, making it look curiously like $Q$ is not negligible in those experimental samples; in several of those works, $Q$ is definitely not negligible and its influence on $S_{\text {eff }}$ is described. There is evidence, however, from alternative experimental techniques that interface state densities can increase with $N_{s}{ }^{18-20}$ Consequently, little can be concluded from experimental trends in $S_{\text {eff }}\left(N_{s}\right)$ without detailed investigation into the experimental samples.
Table I also gives equations for $J_{O s}$ for each special case. The dependence of $J_{O S}$ on $Q$ exhibits the opposite trend to $S_{\text {eff. }}$ That is, when $Q$ is negligible, $J_{O s}$ is inversely proportional to $n_{d}$. And when $Q$ is large, $J_{O S}$ is independent of doping (neglecting any influence on $n_{i s}$ ) and it depends simply on $S_{p 0} / Q^{2}$ or $S_{n 0} / Q^{2}$. This latter dependence can also be construed from (Ref. 2) using Eq. (3).

It is worth noting here that it would be preferable to treat $J_{O s}$ as $J_{O s} /\left(q n_{i e}{ }^{2}\right)$, which is common to all solutions in Table I, and which is measured by standard lifetime experiments before $n_{i e}$ is unnecessarily estimated and extracted. Since the photovoltaics industry is already very familiar with the dimensions and typical magnitudes of $J_{0}$, we do not present our results as $J_{0 s} /\left(q n_{i e}{ }^{2}\right)$ and do not mention it again.

\section{CONDITIONS WHEN $J_{\text {os }}$ AND $S_{\text {eff }}$ ARE INDEPENDENT OF $n_{d}$}

We now determine when the special cases are valid by evaluating the relative error that their underlying assumptions introduce into the minority carrier concentration at the surface. This is equivalent to the relative error in $U_{s}$ for the conditions that give Eq. (7).

To demonstrate the difference between the above special cases, Figure 2 plots the relative error in $p_{s}$ for Cases 1-3 and in $n_{s}$ for Case 4 . They are plotted as a function of band bending $\psi_{s}$ for n-type silicon with $N_{D}=10^{15} \mathrm{~cm}^{-3}$ in low injection at $300 \mathrm{~K}$ using $n_{i e}=10^{10} \mathrm{~cm}^{-3}$ and Boltzmann statistics. Figure 2(a) includes all four cases and Figure 2(b) gives a "close up" of Cases 1-3 for clarity. Note that the doping concentration is only relevant for Case 4 for which the relative error increases with $N_{D}$.

The results of Figure 2 are now compared in terms of the range of $\psi_{s}$ over which the relative error in $p_{s}$ is less than
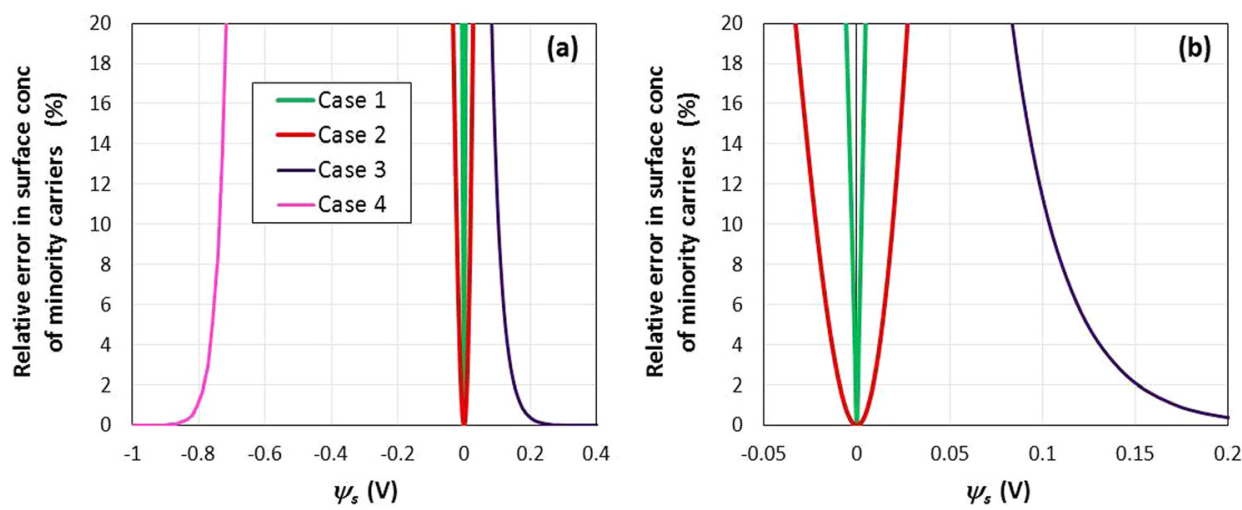

FIG. 2. Relative error in the minority carrier concentration at the surface as a function of the band bending $\psi_{s}$ for the four special cases simulated for an $\mathrm{n}$ type semiconductor. Thus, Cases 1-3 plot the error in $p_{s}$ and Case 4 plots the error in $n_{s}$. Simulations conducted with $N_{D}=10^{15} \mathrm{~cm}^{-3}$, low injection $\left(\Delta n_{d} \ll N_{D}\right), n_{i s}=n_{i d}=10^{10} \mathrm{~cm}^{-3}$, and Boltzmann statistics. Figure (b) displays the results with a smaller $x$-axis range than (a) so that the results for Cases 1-3 are more easily discerned. 
$10 \%$. For Case 1 , this only occurs when $\psi_{s}$ is very close to zero: $-2.72 \mathrm{mV}<\psi_{s}<2.46 \mathrm{mV}$. Hence, band bending must be very small for $S_{\text {eff }}$ to be independent of $n_{d}$. The asymmetry is genuine. It can be shown that for the assumptions contained in Figure 2, the relative error in $p_{s}$ equals $\exp \left(\psi_{s} / V_{T}\right)-1$.

For Case 2, the relative error in $p_{s}$ is less than $10 \%$ when $-22.0 \mathrm{mV}<\psi_{s}<19.3 \mathrm{mV}$. This range is almost 10 times greater than for Case 1. Case 2 therefore provides a useful explicit expression that can be applied in low injection when the charge is small, but it does not mean that $S_{\text {eff }}$ is independent of $N_{D}$.

For Case 3, the valid range is $\psi_{s}>103.7 \mathrm{mV}$. This is an important case. When it holds, $S_{\text {eff }}$ is proportional to $n_{d}$, and $J_{O S}$ is independent of $n_{d}$. It can be shown that the relative error in $p_{s}$ equals $\left[\exp \left(\psi_{s} / V_{T}\right) /\left(\exp \left(\psi_{s} / V_{T}\right)-\left(\psi_{s} / V_{T}\right)-1\right)\right]-1$.

And finally, for Case 4 (strong inversion) the valid range is $\psi_{s}<0.738 \mathrm{~V}$. This limit is strongly dependent on $N_{D}$ (whereas the limit is independent of $N_{D}$ for the first three cases). The dependence on $N_{D}$ is introduced by the $p_{s}$ term on the LHS of Eq. (12). The equation for the relative error in $n_{s}$ is $\left[1+N_{D}{ }^{2} /\right.$ $\left.n_{i}^{2} \times \exp \left(\psi_{s} / V_{T}\right) \times\left(\exp \left(\psi_{s} / V_{T}\right)-\left(\psi_{s} / V_{T}\right)-1\right)\right]^{-1}-1$, which assumes $p_{d}$ is negligible; notice the dependence on $N_{D}$ in this equation.

Thus, the validity of the various special cases, and therefore the relevance of $S_{e f f}$ and $J_{O s}$, depends on $\psi_{s}$, which itself depends on $Q, N_{D}$ and $\Delta n_{d}$.

In Figure 3, we show when each special case is valid at $300 \mathrm{~K}$ for c-Si in low injection as a function of $Q$ and $N_{s}$ for both accumulation and depletion/inversion. We adopt the symbol $N_{s}$ to represent the surface dopant concentration, because the figure is independent of whether the sample is $n$-type or $p$-type. In Figure 3 , the $\mathrm{x}$-axis is $N_{s}$, which is equivalent to $n_{d}$ for $n$-type or $p_{d}$ for $p$-type since low-injection requires $\Delta n_{d} \ll N_{s}$. The results for non-negligible $\Delta n_{d}$ are discussed later with reference to Figure 4.

In Figure 3, the yellow contour shows the range of $Q$ and $N_{s}$ where Case 1 is applicable; i.e., where the approximation that $n_{s}=n_{d}$ and $p_{s}=p_{d}$ is correct to within $10 \%$ error. Thus, at all points shaded yellow in Figure 3, one can reliably assume that in low injection, $S_{\text {eff }}$ equals the minority carrier recombination velocity, either $S_{p 0}$ or $S_{n 0}$ (provided the assumptions entailed in Eq. (7) are valid). One can also reliably assume that $S_{\text {eff }}$ is independent of $N_{s}$ (provided the simplified SRH equation is applicable). These lines are governed by the equations $(Q / q)^{2}=N_{s} \times 1900 \mathrm{~cm}$ for accumulation and $(Q / q)^{2}=N_{s} \times 1600 \mathrm{~cm}$ for depletion.

The red contour shows the range of $Q$ and $N_{D}$ where Case 2 is applicable. At points where $|Q|$ is less than this line (either the red or yellow shaded regions), the approximation that $n_{s}=n_{d} \exp \left[Q / \sqrt{q V_{T} \epsilon_{S i} N_{D}}\right]$ is correct to within $10 \%$ error for c-Si in low injection.

The blue contours indicate the range of $Q$ and $N_{D}$ where Cases 3 and 4 are applicable for accumulation and for inversion, respectively. That is, at all points above the blue line for accumulation, or below the blue line for inversion (shaded in blue), the approximation that either $n_{s}$ or $p_{s}$ equals $Q^{2} / 2 q \epsilon_{S i} V_{T}$ is correct to within $10 \%$ error. In these regions, it can be reliably assumed that $J_{O S}$ is independent of $N_{s}$ (provided the simplified SRH equation is applicable). These lines
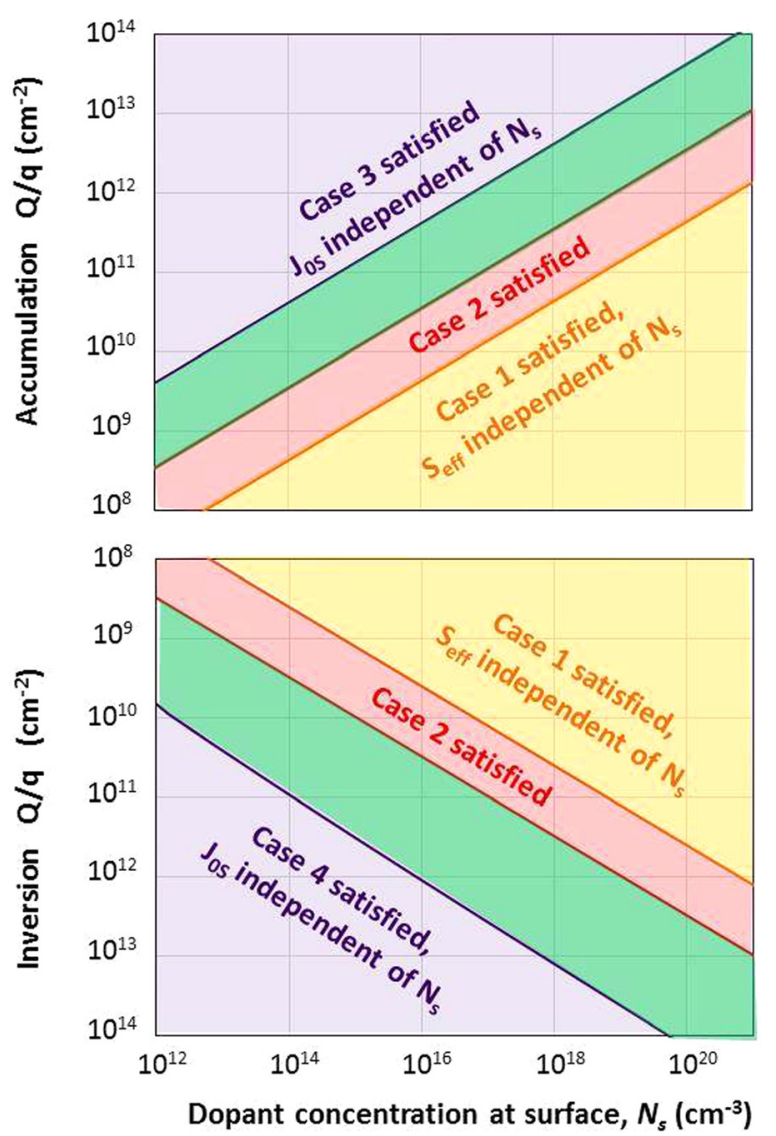

FIG. 3. Contours show where the given assumption represents a $10 \%$ error in the calculation of surface dopant concentration in low injection for c-Si at $300 \mathrm{~K}$; valid for either p-type or n-type c-Si.
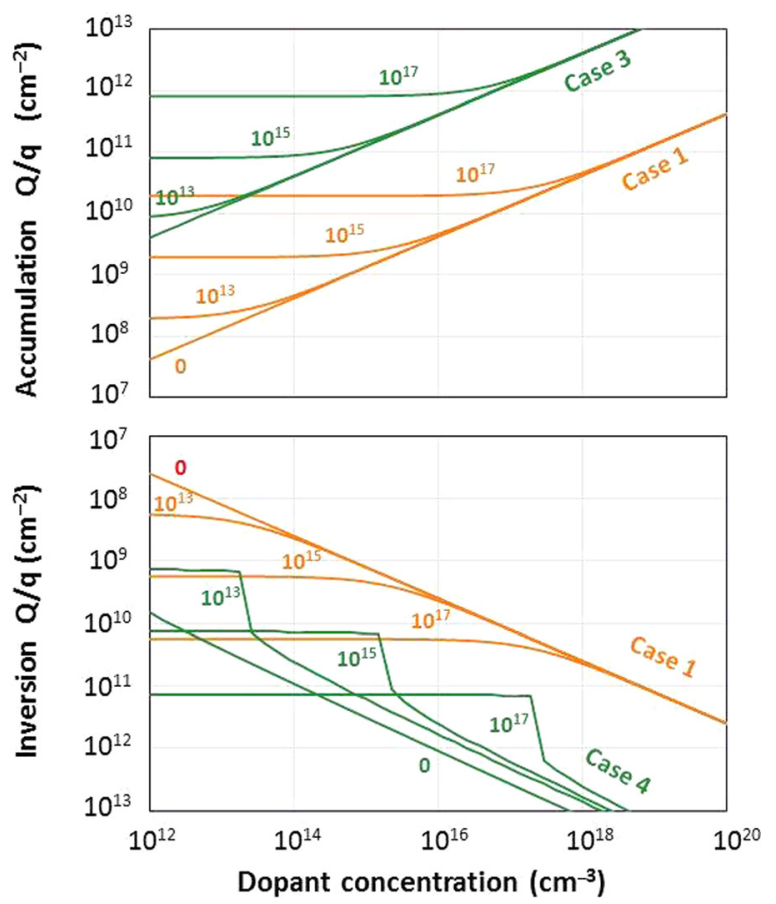

FIG. 4. Contours show where Cases 1, 3, and 4 give a $10 \%$ error in the calculation of the minority carrier surface concentration for c-Si at $300 \mathrm{~K}$; valid for either p-type or n-type c-Si. The contours show $\Delta n_{d}$, the excess carrier concentration in $\mathrm{cm}^{-3}$ at $d$, where the lines for $\Delta n_{d}=0 \mathrm{~cm}^{-3}$ are identical to those shown in Figure 3. $S_{\text {eff }}$ is independent of $N_{s}$ when $|Q|$ is smaller than the orange lines, and $J_{O S}$ is independent of $N_{s}$ when $|Q|$ that is larger than the green lines. 
are governed by the equations $(Q / q)^{2}=N_{s} \times 1.5 \times 10^{7} \mathrm{~cm}$ for accumulation and $(Q / q)^{1.85} \sim N_{s} \times 1.5 \times 10^{6} \mathrm{~cm}$ for inversion.

When comparing accumulation to inversion, there is a slight asymmetry in the yellow and red contours; this asymmetry is smaller when a smaller error limit is applied. By contrast, there is a larger difference between the accumulation and inversion between Case 3 and Case 4, which represent the conditions under which $n_{s}$ and $p_{s}$ are independent of $N_{s}$. In inversion, a significantly higher $Q$ is required to meet the same error limit, where the relative increase in $Q$ increases with $N_{D}$.

Figure 3 shows that for some ranges of $N_{s}$ and $Q$, none of the special cases are justified. This is the region shaded in green. The complicated effects of $Q$ on $U_{s}\left(\Delta n_{\mathrm{d}}\right)$ within this range have been examined elsewhere. ${ }^{1,3,13,21}$

We conclude from Figure 3 that for undiffused c-Si, which typically has $N_{s}<10^{16} \mathrm{~cm}^{-3}$, Case 1 is unjustified unless $|Q|$ is impractically small (less than $5 \times 10^{9} \mathrm{~cm}^{-2}$ ). For diffused c-Si, Case 1 might become valid for dielectrics with a small $|Q|$ when $N_{s}>10^{19} \mathrm{~cm}^{-3}$. To our knowledge, no experimental studies justify the application of Case 1, and it is therefore not recommended that $S_{\text {eff }}$ be assumed equal to $S_{n 0}$ or $S_{p 0}$, or constant with doping for silicon at $300 \mathrm{~K}$.

Figure 3 shows that Case 2 can be used to solve Eq. (12) as an explicit analytical solution over a significantly larger range of $Q$ than Case 1. In practise, it would likely be limited to diffused samples with lowly charged dielectrics.

Figure 3 also shows that Cases 3 and 4 will often be justified for undiffused c-Si. A charge density great than $3 \times 10^{11} \mathrm{~cm}^{-2}$ for accumulation, and $1 \times 10^{12} \mathrm{~cm}^{-2}$ for inversion, is required when $N_{s}<10^{16} \mathrm{~cm}^{-3}$, but these limits decrease as $N_{s}$ decreases. In such case, $J_{O S}$ is independent of $N_{s}$, making it a much more useful effective recombination parameter than $S_{\text {eff. }}$ We also see that very high charge densities are required to justify Cases 3 or 4 for diffused silicon. Recent experiments with $\mathrm{Al}_{2} \mathrm{O}_{3}$ on p-type $\mathrm{Si}$ with $Q / q$ $\sim-2 \times 10^{12} \mathrm{~cm}^{-2}$ clearly showed that Case 3 was unjustified for $N_{s}>10^{18} \mathrm{~cm}^{-3}{ }^{12}$ consistent with Figure 3 .

Finally, we conclude that for diffused silicon and typical charge densities of dielectrics, the most likely scenario is that neither $S_{\text {eff }}$ nor $J_{O S}$ will be independent of $N_{s}$. That is, as $N_{s}$ increases for diffused silicon, $S_{\text {eff }}$ would increase and $J_{O s}$ would decrease, even when $S_{p 0}$ (or $S_{n 0}$ ) are constant.

For simplicity, Figure 3 was restricted to low-injection at $d$; that is $\Delta n_{d} \ll N_{D}$. This condition is readily justified for diffused silicon surfaces. (Note that requiring low-injection at $d$ is a substantially more lenient requirement for diffused surfaces than for undiffused surfaces.)

Figure 4 presents the equivalent situation for several values of $\Delta n_{d}$. In this figure, we do not include the same shading as Figure 3, and we omit the contours for Case 2, which necessarily required low injection. The figure shows that as $\Delta n_{d}$ increases, the range over which Case 1 is valid increases. That is, at small $N_{s}$, Case 1 is valid at higher $Q$ as $\Delta n_{d}$ increases. Nevertheless, it does not change the major conclusion that Case 1 will rarely hold in practical situations. For Case 3, the range of validity decreases, but nevertheless, it should remain valid for most practical cases for undiffused silicon (except for very high $\Delta n_{d}$ ).

\section{EXPERIMENTAL-UNDIFFUSED SILICON}

There are many published examples where the surface recombination of undiffused c-Si is better represented by a $J_{0}$ than an $S_{\text {eff }}$ [e.g., Refs. 22 and 23]. In these cases, $U_{s}$ is proportional to $p_{d} \cdot n_{d}$ rather than to either $p_{d}$ or $n_{d}$ over a wide range of $\Delta n_{d}$. This is equivalent to stating that the surface recombination follows Eq. (2) rather than Eq. (1), and hence, that there is sufficient surface charge for either Case 3 (accumulation) or Case 4 (strong inversion) to be valid.

In Figure 5, we present lifetime measurements that represent Case 4 (strong inversion). The experimental sample is a planar n-type silicon wafer $\left(N_{D}=3.0 \times 10^{15} \mathrm{~cm}^{-3}\right)$ coated with $\mathrm{Al}_{2} \mathrm{O}_{3}$ on both surfaces. Its effective lifetime was measured as a function of $\Delta n_{d}$ by photoconductance using a Sinton Instruments WCT-120 lifetime tool. ${ }^{24}$

Figure 5(a) plots the effective lifetime of the sample before and after the sample was annealed at $425^{\circ} \mathrm{C}$ for $30 \mathrm{~min}$. The anneal caused a significant increase in the effective lifetime, which can be attributed to improved surface passivation by the $\mathrm{Al}_{2} \mathrm{O}_{3}{ }^{12,25}$

Figure 5(b) plots the inverse effective lifetime against $\Delta n_{d}$, where the Auger recombination in the bulk of the wafer has been extracted in the conventional way ${ }^{26}$ using the parameterisation of Richter et $a .^{27}$ As is well documented [e.g., Refs. 26 and 28], a $J_{0}$ of the sample can be extracted from the slope of the data in Figure 5(b) provided that the slope is constant over a wide range of $\Delta n_{d}$. This provision is satisfied for the pre-annealed sample, for which $J_{O S}=12 \mathrm{fA} / \mathrm{cm}^{2}$. By applying the appropriate equation in Table $\mathrm{I}, J_{O S}$ can then be converted to $S_{p o} / Q^{2}=8.6 \times 10^{16} \mathrm{~cm}^{4} \mathrm{C}^{-2} \mathrm{~s}^{-1}$.

For the post-annealed sample, the slope of the data in Figure 5(b) is clearly not straight. Consequently, $J_{O s}$ cannot
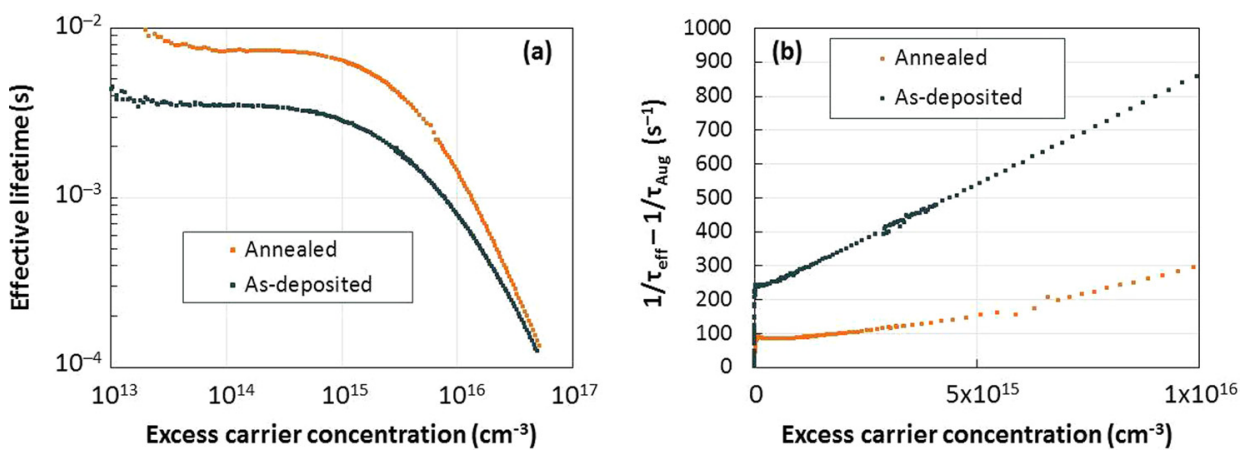

FIG. 5. Photoconductance lifetime measurements on undiffused $n$-type silicon passivated with $\mathrm{Al}_{2} \mathrm{O}_{3}$ before and after an anneal, plotting (a) effective lifetime against $\Delta n_{d}$, and (b) the same data presented as the inverse effective lifetime and where Auger recombination in the bulk has been extracted. 
be reliably extracted without additional information about the sample. The most likely cause for this feature is that the bulk recombination is neither negligible nor constant with $\Delta n_{d}$.

From this example-and several others in the literature [e.g., Refs. 22 and 23] —it is evident that $J_{O S}$ can be directly determined from lifetime measurements on non-diffused samples that have a high surface charge (provided that bulk recombination is either negligible or constant with $\Delta n_{d}$ ).

\section{EXPERIMENTAL-DIFFUSED SILICON}

Lifetime measurements are routinely employed to determine the recombination current of heavily doped layers $J_{0 e}$ (e.g., an emitter or a back-surface field). The $J_{0 e}$ depends on the SRH recombination at the surface, and on the SRH and Auger recombination within the layer. The contributions to the $J_{O e}$ made by these mechanisms,

$$
J_{0 e}=J_{0 e, \text { surface SRH }}+J_{0 e, S R H}+J_{0 e, \text { Auger }},
$$

can be determined by a careful simulation of the heavily doped layer. ${ }^{15,29-31}$ This requires consideration of carrier mobility, band-gap narrowing, Fermi-dirac statistics, interface properties, and the Auger coefficients. The parameters in Eq. (20) can be determined directly with EDNA 2 (Refs. 31 and 32) or construed from the outputs of device simulators such as PC1D ${ }^{33}$ and Sentaurus, ${ }^{34}$ or from various analytical approaches. ${ }^{29}$

In Figure 6(a), we re-plot the recent results of Ref. 12 to show $J_{O e}, J_{O e, \text { surface } S R H}$ and $J_{O e, \text { Auger }}$ for 21 samples, where the X-axis label states the boron surface concentration $N_{s}$. $J_{O e, S R H}$ is assumed negligible. ${ }^{12}$ These results were derived from planar c-Si wafers diffused with boron and coated with $\mathrm{Al}_{2} \mathrm{O}_{3}$. The figure shows that the contribution to $J_{0 e}$ from surface recombination is relatively constant with surface concentration; by contrast, the contribution from Auger recombination increases substantially and this is the reason that $J_{O e}$ increases with $N_{s}$.

In Figure 6(b), we plot $S_{e f f}$ and $J_{O s}$ against $N_{s}$, where the simulation with EDNA 2 is used to determine $S_{\text {eff }}$ via Eq. (1) and $J_{O S}$ via Eq. (2). The symbols represent the experimental diffused (circles) and undiffused (squares) $p$-type samples. The lines in Figure 6(b) are simulated from the interface parameters determined by $\mathrm{CV}$ measurements on undiffused samples, as described in detail in Ref. 12.

Figure 6(b) shows that $S_{\text {eff }}$ increases with $N_{s}$, as has often been observed in studies on heavily doped silicon. ${ }^{5-12}$ (Exceptions to this trend also exist ${ }^{35,36}$ ). It would be wrong to conclude, however, that the reason that $S_{\text {eff }}$ increases with $N_{s}$ is necessarily because $Q$ decreases or $S_{n 0}$ increases (e.g., by an increase in interface defects). It was shown in Ref. 12 that the dependence of $J_{O e}$ on $N_{s}$ presented in Figure 6(b) is entirely consistent with a constant $S_{n 0}$ of $1.1 \times 10^{4} \mathrm{~cm} / \mathrm{s}$ and a constant $Q / \mathrm{q}$ of $-1.7 \times 10^{12} \mathrm{~cm}^{-2}$. Thus, the increase in $S_{\text {eff }}$ observed in Figure 6(b) is an artefact of its derivation, as explained in Sec. V. In short, the surface charge is too large for Case 1 to be satisfied, and thus $S_{\text {eff }}$ necessarily depends on $N_{s}$. Put otherwise, $N_{s}$ and $Q$ do not fall within the yellow region of Figure 3.
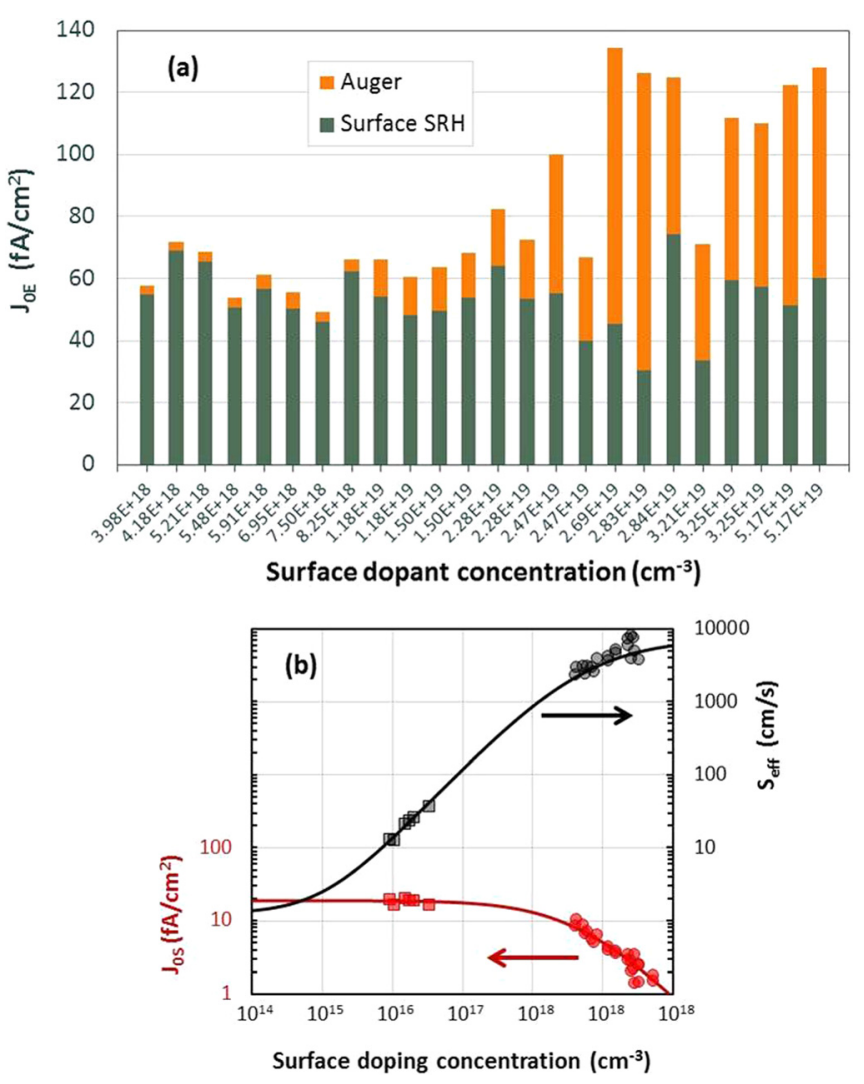

FIG. 6. Photoconductance lifetime measurements of $\mathrm{Al}_{2} \mathrm{O}_{3}$-coated $\mathrm{Si}$ wafers presented in Ref. 12 replotted to show (a) experimental $J_{0 e}$ and calculated $J_{O e, \text { surface } S R H}$ and $J_{O e, \text { layer Auger }}$, and (b) $S_{\text {eff }}$ and $J_{O s}$ against the boron surface concentration.

In summary, plots of $S_{\text {eff }}$ against $N_{s}$ are easily misconstrued. Here, the example illustrates how $S_{\text {eff }}$ can increase with $N_{s}$ when all interface parameters are constant with $N_{s}$. We do not discount the possibility that in some structures, interface parameters could change with $N_{s}$, as has been concluded by other experimental techniques, ${ }^{18-20}$ but it is clear that changes in interface parameters cannot be determined from $S_{\text {eff }}\left(N_{s}\right)$ without accounting for the influence of $Q, N_{s}$, $\Delta n_{d}$, and the assumptions entailed in Eq. (7). Moreover, when $N_{s}$ is high, analyses need also account for uncertainties in the physical models for band-gap narrowing and Auger recombination (which are currently rather large), as well as variations in $n_{i e}$ between $x=s$ and $x=d$ as outlined in Appendix A.

Finally, Figure 6(b) plots $J_{O s}$ vs $N_{s}$, as determined by Eq. (2). At $N_{s}<10^{17} \mathrm{~cm}^{-3}, J_{O s}$ is approximately constant with $N_{s}$, indicative of $Q$ being sufficiently large relative to $N_{s}$ that Case 3 is satisfied (i.e., the samples are within the blue region of Figure 3). In this range, $J_{0 s}$ is a particularly useful parameter because it provides a single value to quantify the surface passivation and is independent of the substrate properties $\left(N_{s}\right.$ and $\left.\Delta n_{d}\right)$.

At $N_{s}>10^{17} \mathrm{~cm}^{-3}, J_{O s}$ decreases with $N_{s}$. Over this range, $J_{O s}$ is as flawed as $S_{e f f}$ in quantifying the surface passivation, because neither parameter defines the quality of the surface passivation independently of the substrate properties. 


\section{CONCLUSION}

This paper described two effective surface recombination parameters, $S_{\text {eff }}$ and $J_{O s}$. Their dependence on interface parameters and dopant surface concentration $N_{s}$ was derived. It was found that for c-Si at $300 \mathrm{~K}$ in low-injection, $S_{\text {eff }}$ is independent of $N_{s}$ only when $Q^{2} / N_{s}<1900 \mathrm{~cm}$ for accumulation and $<1600 \mathrm{~cm}$ for depletion; otherwise $S_{\text {eff }}$ increases with $N_{s}$. This condition would be rarely satisfied in undiffused wafers but sometimes satisfied in heavily diffused wafers (provided they are coated with lowly charged films). Also for c-Si at $300 \mathrm{~K}$ in low-injection, $J_{O S}$ is independent of $N_{s}$ when $Q^{2} / N_{s}$ $>1.5 \times 10^{7} \mathrm{~cm}$ for accumulation and $Q^{1.85} / N_{s}>1.5 \times 10^{6} \mathrm{~cm}$ for inversion. These conditions are commonly satisfied in undiffused wafers but rarely in diffused wafers. Thus, $J_{O S}$ is a superior metric for comparing surface passivation of undiffused samples than the current convention of comparing $S_{\text {effi }}$; nevertheless, the derivation and limitations of $J_{0 s}$ should be understood before its widespread application.

\section{APPENDIX A: EQUATIONS WHEN $p_{\text {os }} n_{\text {Os }}$ DOES NOT EQUAL $p_{\text {od }} n_{\text {od }}$}

The equations in the main body of this paper were derived under the assumption that $p_{O S} n_{O s}=p_{0 \mathrm{~d}} n_{0 \mathrm{~d}}$; that is, that the effects of BGN and degeneracy are identical at $s$ and $d$. This appendix states how the equations would be reexpressed without this assumption. We now use $n_{i s}{ }^{2}$ to represent $p_{O S} n_{O s}$ and $n_{i d}{ }^{2}$ to represent $p_{0 \mathrm{~d}} n_{0 \mathrm{~d}}$.

In Eqs. (4)-(6) and (11), as well as all equations for $J_{O e}$ in Table I, $n_{i e}$ should be replaced with $n_{i s}$.

Equations for $S_{\text {eff }}$ in Table I (except Case 1) should be multiplied by the factor $n_{i s}^{2} / n_{i d}^{2}$.

Equations (13)-(15) should be rewritten

$$
\begin{gathered}
n_{s}=\frac{n_{i s}}{n_{i d}} n_{d} \exp \left(\frac{\psi_{s}}{V_{T}}\right), \\
p_{s}=\frac{p_{i s}}{p_{i d}} p_{d} \exp \left(-\frac{\psi_{s}}{V_{T}}\right), \text { and } \\
\frac{p_{s} n_{s}}{n_{i s}^{2}}=\frac{p_{d} n_{d}}{n_{i d}^{2}} .
\end{gathered}
$$

\section{APPENDIX B: THE DEPENDENCE OF $S_{p o}$ AND $S_{n o}$ ON ENERGY-DEPENDENT DEFECTS}

Defect states at a semiconductor surface do not have the same energy at all spatial locations. Instead, there is a distribution of defect energies introduced by variable bond strain. The capture cross sections are also often assumed to vary with the energy of the defect. ${ }^{1}$ To account for these energy dependences when determining $U_{s}$, an approach that integrates the SRH equation as a function of energy is often applied, for which the energy-dependent variables are $n_{1}, p_{1}, D_{i t}, \sigma_{p}$, and $\sigma_{p}$. When this is the case, the SRH equation is ${ }^{1}$

$$
U_{s}=\left(p_{s} n_{s}-n_{i s}{ }^{2}\right) \int_{E_{v}}^{E_{c}} \frac{d E}{\frac{p_{s}+p_{1}(E)}{S_{n 0}(E)}+\frac{n_{s}+n_{1}(E)}{S_{p 0}(E)}},
$$

where $S_{n 0}(E)=v_{t h n} D_{i t}(E) \sigma_{n}(E)$ and $S_{p 0}(E)=v_{t h p} D_{i t}(E) \sigma_{p}(E)$.
The principle problem with solving Eq. (B1) is that it requires an intimate knowledge of the interface. That is, for any given passivation scheme and sample, researchers must determine many energy-dependent values in order to determine $U_{s}$ from its interface parameters.

When four particular requirements are satisfied, however, a far simpler relationship between $U_{s}$ and the interface parameters can be derived. The first requirement is that $n_{1}$ and $p_{1}$ be negligible, as occurs when there is negligible recombination through defects with energies near the band edge. This requirement is met by $\mathrm{Si}-\mathrm{SiO}_{2}$ (Ref. 37) and $\mathrm{Si}-\mathrm{Al}_{2} \mathrm{O}_{3} \cdot{ }^{38}$ The second requirement is that $\sigma_{p}(E)$ and $\sigma_{p}(E)$ exhibit a negligible dependence on $E$ for each defect. Careful experimentation suggests that this is a reasonable approximation for $\mathrm{Si}-\mathrm{SiO}_{2}$ (Refs. 39-43) and $\mathrm{Si}-\mathrm{Al}_{2} \mathrm{O}_{3}{ }^{38,12}$ The third requirement is that the energy distribution of the defect can be represented by a Gaussian distribution

$$
D_{i t}(E)=D_{\text {itmax }} \exp \left[-\left(\frac{E-E_{\max }}{\sqrt{2} \mu}\right)^{2}\right],
$$

where $D_{\text {it max }}$ and $E_{\max }$ are the $D_{i t}$ and energy at the peak of the distribution, and $\mu$ is the standard deviation of the distribution. (In fact, any distribution whose integral between $E_{v}$ and $E_{c}$ is approximately the same as the integral between $-\infty$ and $+\infty$ will do.) The fourth and final requirement-as was imposed in the main body of the paper-is that only one of the denominator terms in the SRH equation is significant.

When satisfied, these four simplifications modify Eq. (B1) to give

$$
U_{s}=p_{s} S_{p 0}
$$

where

$$
S_{p 0}=v_{t h} \sigma_{p} D_{\text {itmax }} \int_{E_{v}}^{E_{c}} \exp \left[-\left(\frac{E-E_{\text {max }}}{\sqrt{2} \mu}\right)^{2}\right] d E,
$$

which for a strongly peaked distribution, solves to give

$$
S_{p 0}=\sqrt{2 \pi} v_{t h} \sigma_{p} D_{i t \max } \mu .
$$

An analogous equation can be derived for $S_{n 0}$.

The principle value of Eq. (B5) lies in its simplicity. It states that the recombination is independent of defect energy $E$ and can be computed without numerical integration. Moreover, for a given defect type, where one might expect $\sigma_{p}$ and $\mu$ to vary little from sample to sample, one could state that

$$
S_{p 0}=c_{\text {defect }} D_{\text {itmax }},
$$

where $c_{\text {defect }}$ is a constant that represents a type of defect, such as the $P_{b}$ bond at the $\mathrm{Si}-\mathrm{SiO}_{2}$ interface, ${ }^{44}$ or the principle donor-like bond at the $\mathrm{Si}-\mathrm{Al}_{2} \mathrm{O}_{3}$ interface. ${ }^{38}$ If this proves viable, then it enables a simpler comparison between passivation schemes, deposition technologies, and annealing conditions. For a given interface, once $c_{\text {defect }}$ is determined 
for one passivation scheme, one need only determine $D_{\text {it } \max }$ to calculate the recombination velocity of any other substrate passivated by the same scheme.

We emphasise that Eq. (B6) entails several assumptions and should only be applied after they have been appropriately assessed.

\section{APPENDIX C: EXPLICIT SOLUTIONS TO EQUATION (12) WHEN THE SURFACE IS IN WEAK DEPLETION OR ACCUMULATION}

We can find explicit solutions to Eq. (12) for the condition where the majority carrier concentration is far greater than the minority carrier concentration at both $s$ and $d$. This is valid from weak depletion to strong accumulation. In the case of an $n$-type semiconductor, this requires $n_{s}, n_{d} \gg p_{s}$, $p_{d}$, simplifying Eq. (12) to give

$$
\exp \left(\frac{\psi_{s}}{V_{T}}\right)-1-\frac{N_{D}}{n_{d}} \frac{\psi_{s}}{V_{T}}=\frac{Q^{2}}{2 q \epsilon_{S i} V_{T} n_{d}} .
$$

In low injection, when $n_{d} \sim N_{D}$, it can be further simplified by noting that the LHS is a partial Maclaurin series

$$
\sum_{k=2}^{\infty} \frac{\left(\psi_{s} / V_{T}\right)^{k}}{k !}=\frac{Q^{2}}{2 q \epsilon_{s} V_{T} N_{D}} .
$$

In Case $1, Q$ is sufficiently small that $\psi_{s}$ is negligible; in Case 2, $Q$ is sufficiently small that only the $k=2$ term is significant and the LHS of Eq. (C1) equals $\left(\psi_{S} / V_{T}\right)^{2} / 2$; in Cases 3 and 4, the LHS of Eq. (C1) is assumed equal to $\exp \left(\psi_{S} / V_{T}\right)$.

For p-type, $N_{D}$ in Eq. (C2) is replaced by $N_{A}$.

${ }^{1}$ A. G. Aberle, Crystalline Silicon Solar Cells: Advanced Surface Passivation and Analysis (University of New South Wales, Sydney, 1999), Chap. 2.

${ }^{2}$ B. Kuhlmann, "Charakterisierung und mehrdimensionale Simulation von MIS-Inversionsschichtsolarzellen," Ph.D. Thesis (University of Hannover, Germany, 1998), p. 71.

${ }^{3}$ J. Brody and A. Rohatgi, "Analytical approximation of effective surface recombination velocity of dielectric-passivated p-type silicon," Solid-State Electron. 45, 1549-1557 (2001).

${ }^{4}$ S. Steingrube, P. P. Altermatt, D. S. Steingrube, J. Schmidt, and R. Brendel, "Interpretation of recombination at $\mathrm{c}-\mathrm{Si} / \mathrm{SiN}_{\mathrm{x}}$ interfaces by surface charge," J. Appl. Phys. 108, 014506 (2010).

${ }^{5}$ R. R. King, R. A. Sinton, and R. M. Swanson, IEEE Trans. Electron Devices 37, 365 (1990).

${ }^{6}$ A. Cuevas, P. A. Basore, G. Giroult-Matlakowski, and C. Dubois, J. Appl. Phys. 80, 3370 (1996).

${ }^{7}$ A. Cuevas, M. Stuckings, J. Lau, and M. Petravic, in Proceedings of the 14th European Photovoltaic Solar Energy Conference, Barcelona (HS Stephens, Falmerston, UK, 1997), p. 2416.

${ }^{8}$ P. P. Altermatt, J. Schumacher, A. Cuevas, M. J. Kerr, S. W. Glunz, R. R. King, G. Heiser, and A. Schenk, "Numerical modeling of highly doped Si:P emitters based on Fermi-Dirac statistics and self-consistent material parameters," J. Appl. Phys. 92, 3187-3197 (2002).

${ }^{9}$ A. Cuevas, M. Kerr, and J. Schmidt, in Proceedings of the 3rd World Conference on Photovoltaic Energy Conversion, Osaka (2003), p. 913.

${ }^{10}$ P. P. Altermatt, H. Plagwitz, R. Bock, J. Schmidt, R. Brendel, M. J. Kerr, and A. Cuevas, "The surface recombination velocity at boron-doped emitters: comparison between various passivation techniques," in Proceedings of the 21st European Photovoltaic Solar Energy Conference, Dresden, Germany (WIP, Munich, 2006), p. 647.

${ }^{11}$ B. Hoex, J. Schmidt, R. Bock, P. P. Altermatt, M. C. M. van de Sanden, and W. M. M. Kessels, "Excellent passivation of highly doped p-type Si surfaces by the negative-charge-dielectric $\mathrm{Al}_{2} \mathrm{O}_{3}$, , Appl. Phys. Lett. 91, 112107 (2007).

${ }^{12}$ L. E. Black, T. Allen, K. R. McIntosh, and A. Cuevas, "Effect of boron concentration on recombination at the $\mathrm{p}-\mathrm{Si}-\mathrm{Al}_{2} \mathrm{O}_{3}$ interface," J. Appl. Phys. 115, 093707 (2014).

${ }^{13}$ A. G. Aberle, S. Glunz, and W. Warta, "Impact of illumination level and oxide parameters on Shockley-Read-Hall recombination at the $\mathrm{Si}-\mathrm{SiO}_{2}$ interface," J. Appl. Phys. 71(9), 4422-4431 (1992).

${ }^{14}$ S. J. Robinson, S. R. Wenham, P. P. Altermatt, A. G. Aberle, G. Hesier, and M. A. Green, "Recombination rate saturation mechanisms at oxidized surfaces of high-efficiency silicon solar cells," J. Appl. Phys. 78, 4740-4754 (1995).

${ }^{15}$ J. A. del Alamo and R. M. Swanson, "The physics and modelling of heavily doped emitters," IEEE Trans. Electron Devices 31, 1878-1888 (1984).

${ }^{16}$ R. B. M. Girisch, R. P. Mertens, and R. F. De Keersmaecker, "Determination of $\mathrm{Si}_{-} \mathrm{SiO}_{2}$ interface recombination parameters using a gate-controlled point-junction diode under illumination," IEEE Trans. Electron Devices 35, 203-222 (1988).

${ }^{17}$ A. S. Grove and D. J. Fitzgerald, "Surface effects on $\mathrm{p}-\mathrm{n}$ junctions: Characteristics of surface space-charge regions under non-equilibrium conditions," Solid-State Electron. 9, 783-806 (1966).

${ }^{18}$ J. Snel, Solid-State Electron. 24, 135 (1981).

${ }^{19}$ M. Y. Ghannam, R. P. Mertens, R. F. De Keersmaecker, and R. J. van Overstraeten, IEEE Trans. Electron Devices 32, 1264 (1985).

${ }^{20}$ H. Jin, W. E. Jellett, Z. Chun, K. J. Weber, A. W. Blakers, and P. J. Smith, Appl. Phys. Lett. 92, 122109 (2008).

${ }^{21}$ Z. Hameiri, K. McIntosh, and G. Xu, "Evaluation of recombination processes using the local ideality factor of carrier lifetime measurements," Sol. Energy Mater. Sol. Cells 117, 251-258 (2013).

${ }^{22} \mathrm{~W}$. E. Jellet and K. J. Weber, "Accurate measurement of extremely low surface recombination velocities on charged, oxidized silicon surfaces using a simple metal-oxide-semiconductor structure," J. Appl. Phys. 90, 042104 (2007).

${ }^{23}$ W. Liang, K. J. Weber, D. Suh, S. Phang, J. Yu, A. K. McAuley, and B. R. Legg, "Surface passivation of boron-diffused p-type silicon surfaces with (100) and (111) orientations by $\mathrm{ALD} \mathrm{Al}_{2} \mathrm{O}_{3}$ layers," IEEE J. Photovoltaics 3, 678-683 (2013).

${ }^{24}$ See http://www.sintoninstruments.com/ for a description of the WCT-120 lifetime tool (last accessed 4 April 2014).

${ }^{25}$ L. E. Black and K. R. McIntosh, "Surface passivation of c-Si by atmospheric pressure chemical vapour deposition of $\mathrm{Al}_{2} \mathrm{O}_{3}$," Appl. Phys. Lett. 100, 202107 (2012).

${ }^{26}$ D. E. Kane and R. M. Swanson, in Proceedings of the 18th IEEE Photovoltaic Specialists Conference, Las Vegas, 1985 (IEEE, 1985), p. 578.

${ }^{27}$ A. Richter, S. W. Glunz, F. Werner, J. Schmidt, and A. Cuevas, Phys. Rev. B 86, 165202 (2012).

${ }^{28} \mathrm{H}$. Mäckel and K. Varner, "On the determination of the emitter saturation current density from lifetime measurements of silicon devices," Prog. Photovoltaics 21, 850-866 (2012).

${ }^{29} \mathrm{~A}$. Cuevas and M. A. Balbuena, "Review of analytical models for the study of highly doped regions of silicon devices," IEEE Trans. Electron Devices 36, 553-560 (1989).

${ }^{30}$ A. Cuevas, R. Merchán, and J. C. Ramos, "On the systematic analytical solutions for minority-carrier transport in nonuniform doped semiconductors: Application to solar cells," IEEE Trans. Electron Devices 40, 1181-1183 (1993).

${ }^{31}$ K. R. McIntosh and P. P. Altermatt, "A freeware 1D emitter model for silicon solar cells," in Proceedings of the 35th IEEE Photovoltaic Specialists Conference, Honolulu (2010), pp. 2188-2193.

${ }^{32}$ K. R. McIntosh, P. P. Altermatt, T. J. Ratcliff, K. C. Fong, L. E. Black, S. C. Baker-Finch, and M. D. Abbott, "An examination of three common assumptions used to simulate recombination in heavily doped silicon," in Proceedings of the 28th EU PVSEC, Paris (2013), pp. 1672-1679.

${ }^{33}$ D. A. Clugston and P. A. Basore, "PC1D version 5: 32-bit solar cell modelling on personal computers," in Proceedings of the 26th IEEE Photovoltaic Specialists Conference, Anaheim (1997), pp. 207-210.

${ }^{34}$ See www.synopsys.com/products/tcad/tcad.html. for SENTAURUS, Synopsys Inc. Mountain View, CA (last accessed, 6 March 2014).

${ }^{35}$ R. R. King, R. A. Sinton, and R. M. Swanson, "Studies of diffused boron emitters: Saturation current, bandgap narrowing and surface recombination velocity,” IEEE Trans. Electron Devices 38, 1399-1409 (1991).

${ }^{36}$ F. Ma, S. Duttagupta, M. Peters, G. S. Samudra, A. G. Aberle, and B. Hoex, "Numerical analysis of $\mathrm{p}+$ emitters passivated by a PECVD AlOx/ SiNx stack," Energy Procedia 38, 124-130 (2013).

${ }^{37}$ W. Füssel, M. Schmidt, H. Angermann, G. Mende, and H. Flietner, Nucl. Instrum. Methods Phys. Res. A 377, 177 (1996). 
${ }^{38}$ L. E. Black and K. R. McIntosh, "Modeling recombination at the $\mathrm{Si}-\mathrm{Al}_{2} \mathrm{O}_{3}$ interface," IEEE J. Photovoltaics 3(3), 936-943 (2013).

${ }^{39} \mathrm{~W}$. Fahrner and A. Goetzberger, "Energy dependence of electrical properties of interface states in $\mathrm{Si}_{-} \mathrm{SiO}_{2}$ interfaces," Appl. Phys. Lett. 17, 16-18 (1970).

${ }^{40}$ J. A. Cooper and R. J. Schwartz, "Electrical characteristics of the $\mathrm{SiO} 2-\mathrm{Si}$ interface near midgap and in weak inversion," Solid-State Electron. 17, 641-654 (1974).

${ }^{41}$ H. Deuling, E. Klausmann, and A. Goetzberger, "Interface states in Si-SiO2 interfaces," Solid-State Electron. 15, 559-571 (1972).
${ }^{42}$ M. Morita, K. Tsubouchi, and N. Mikoshiba, "Measurement of interface parameters near the band edge at the $\mathrm{Si} / \mathrm{SiO} 2$ interface by the conductance method," Appl. Phys. Lett. 33, 745-747 (1978).

${ }^{43}$ M. El-Sayed, G. Pananakakis, and G. Kamarinos, "Complete exploration of the silicon gap at the $\mathrm{Si}-\mathrm{SiO} 2$ interface of MIS tunnel diodes using the conductance technique at various temperatures and illumination levels," Solid-State Electron. 28, 345-357 (1985).

${ }^{44} \mathrm{~A}$. Stesmans, "Passivation of $\mathrm{Pb} 0$ and $\mathrm{Pb} 1$ interface defects in thermal (100) $\mathrm{Si} / \mathrm{SiO}_{2}$ with molecular hydrogen," Appl. Phys. Lett. 68(15), 2076-2078 (1996). 